\title{
Die Geschichte der deutschen Europawissenschaften im Arbeitskreis Europäische Integration
}

\author{
Frédéric Krumbein ${ }^{*}$
}

Der Arbeitskreis Europäische Integration e.V. (AEI) ist die älteste und größte interdisziplinäre europawissenschaftliche Vereinigung in Deutschland und eine der größten und aktivsten weltweit.

Von seinem Selbstverständnis her ist der AEI eine unabhängige Vereinigung, deren Ziel die wissenschaftliche Analyse und Diskussion der europäischen Integration darstellt. Zu diesem Zweck organisiert der Arbeitskreis wissenschaftliche Tagungen, publiziert eine europawissenschaftliche Schriftenreihe und Fachzeitschrift und fördert insgesamt die Vernetzung und den Austausch der Europawissenschaftlerinnen und Europawissenschaftler in Deutschland und Europa. Es gehört zu den Zielen des AEI, Orientierungspunkte und Impulse für den Prozess der europäischen Integration zu geben.

Darüber hinaus legt der AEI Wert auf eine enge Anbindung an die europapolitische Praxis durch die Einbeziehung von thematisch im EU-Bereich tätigen Praktikerinnen und Praktikern als Mitglieder und Vortragende bei Tagungen. Weitere Charakteristika sind die Interdisziplinarität der Vereinigung, insbesondere durch die Einbindung der Politik-, Rechts- und Wirtschaftswissenschaften, sowie die Förderung des wissenschaftlichen Nachwuchses.

\section{Die Anfänge einer interdisziplinären wissenschaftlichen Vereinigung}

In der Anfangszeit der europäischen Integration wünschten sich die Europäische Kommission und ihr Verbindungsbüro in Deutschland eine engere Kooperation mit Universitäten und Forschungseinrichtungen. Seit 1964 gab es deshalb eine Abteilung für wissenschaftliche Information im Bonner Verbindungsbüro der Kommission, um die Forschung und Lehre zu europäischen Themen in der deutschen Hochschullandschaft zu fördern. Die Informationstätigkeit des Verbindungsbüros stieß auf ein positives Echo innerhalb der deutschen Wissenschaft. Das Verbindungsbüro gründete daraufhin im Jahr 1966 zusammen mit einer Gruppe von WissenschaftlerInnen und PraktikerInnen, die im Bereich der europäischen Integration tätig waren, einen nicht rechtsfähigen Verein mit der Bezeichnung „Europäische Gemeinschaften, Arbeitskreis für Europakunde“.

Am 12. Dezember 1969 wurde dieser Arbeitskreis in einen rechtsfähigen Verein mit der bis heute bestehenden Bezeichnung „Arbeitskreis Europäische Integration e.V.“ umgegründet. Bei der Gründungsversammlung waren 24 Personen anwesend, die anschließend allesamt in den Vorstand beziehungsweise in das Präsidium gewählt wurden. Der AEI wurde am 9. März 1970 in das Vereinsregister in Bonn eingetragen. Zu den Gründungsmitgliedern gehörten der erste Präsident der EG-Kommission, Walter Hallstein, und der Vordenker der sozialen Marktwirtschaft und Staatssekretär für europäische Angelegenheiten im Bundeswirtschaftsministerium, Alfred Müller-Armack.

Die Organisationsstruktur des Arbeitskreises wurde von den Gründervätern und -müttern an die Struktur eines Unternehmens angelehnt. Die Mitgliederversammlung als oberstes Entschei-

* Dr. Frédéric Krumbein, Geschäftsführer des Arbeitskreises Europäische Integration, Berlin. 
dungsgremium tritt regulär einmal im Jahr zusammen. Sie entscheidet im Wesentlichen über Satzungsänderungen, entlastet den Vorstand und wählt das Präsidium für drei Jahre. Das Präsidium bildet den ,Aufsichtsrat" des Arbeitskreises und wählt wiederum den Vorstand für eine Amtszeit von drei Jahren. Der Vorstand beruft eine Geschäftsführung. Vorstand und Geschäftsführung erledigen die tägliche Arbeit der Vereinigung zur Erfüllung der satzungsgemäßen Ziele und repräsentieren den Arbeitskreis nach außen. ${ }^{1}$

Die Ziele des AEI lauten gemäß seiner Satzung wie folgt: „Der Arbeitskreis Europäische Integration verfolgt das Ziel, Informationen über die Europäischen Gemeinschaften im Bereich der Universitäten und Hochschulen zu vermitteln und die wissenschaftliche Forschung auf dem Gebiet der europäischen Einigung zu fördern. Diesem Ziel sollen Tagungen, Vortragsveranstaltungen und Veröffentlichungen sowie Austausch und Registrierung von Dokumenten und einschlägigen wissenschaftlichen Arbeiten dienen.“

\section{Analyse, Diskussion und Orientierung der europäischen Integration}

Kern der Aktivitäten des AEI sind seine wissenschaftlichen Tagungen, aus denen Fachbeiträge und Bücher hervorgehen. Der Arbeitskreis hat seit seiner Gründung mindestens 339 wissenschaftliche Konferenzen mit über 17.500 Teilnehmerinnen und Teilnehmern veranstaltet. Im Schnitt hat der AEI damit sieben bis acht Konferenzen pro Jahr veranstaltet. Sechs Konferenzen waren das Minimum, zwölf das Maximum. ${ }^{2}$

Von Anfang an war es das Ziel, sowohl interdisziplinäre als auch fachspezifische Tagungen zu veranstalten, den wissenschaftlichen Nachwuchs im Besonderen anzusprechen und den Dialog mit der Praxis zu suchen. Bereits im ersten Tätigkeitsbericht des Arbeitskreises werden als Tagungsarten folgende genannt: „1) allgemeine Informationstagungen über den Stand der europäischen Integration und die aktuellen Probleme der Europäischen Gemeinschaften, mit denen Interessenten aller Disziplinen angesprochen werden; 2) Fachtagungen für Juristen, Wirtschaftswissenschaftler, Politologen [...]; 3) interdisziplinäre Assistentenseminare, in denen ein Problemkreis aus dem Bereich der Integrationspolitik in seinen verschiedenen Teilaspekten aus der Betrachtung der verschiedenen Disziplinen erörtert wird; 4) wissenschaftliche Kolloquien, in denen namhafte Wissenschaftler die Möglichkeit zum Gedankenaustausch untereinander, aber auch zu einem Dialog mit Mitgliedern und höheren Beamten der Kommission der Europäischen Gemeinschaften, Vertretern des Ministerrates, des Europäischen Gerichtshofes und des Europäischen Parlaments haben."

Die drei Disziplinen Politik-, Rechts- und Wirtschaftswissenschaften stellten von Anfang an die größten Teilnehmergruppen an den Tagungen des AEI. In den 1970er Jahren waren dabei die Rechts- und Wirtschaftswissenschaften noch etwas stärker vertreten als die Politikwissenschaft. Inzwischen ist das Verhältnis zwischen den drei Disziplinen ausgewogen.

Etwa ein Drittel der Tagungen fand in den jeweiligen deutschen Regierungssitzen Bonn und später Berlin statt. Die beiden Konferenzorte wurden auch deshalb so oft ausgewählt, weil viele Akteure aus der europapolitischen Praxis als Vortragende und Teilnehmerinnen und Teilnehmer zu Tagungen in Bonn und Berlin kommen konnten. Bei der Wahl der Tagungsorte spielten, neben der Nähe zu den Akteuren aus der politischen Praxis und der geografischen Verteilung, auch

1 Für eine Übersicht über die Präsidenten, Vorstandsvorsitzenden und Geschäftsführer des AEI siehe Anhang I.

2 Für eine Übersicht über die vom AEI organisierten Konferenzen von 1969 bis 2013 siehe Anhang II. 
immer der finanzielle und organisatorische Beitrag der Kooperationspartner vor Ort eine wichtige Rolle.

Die internationale Ausrichtung des Arbeitskreises zeigt sich unter anderem darin, dass 23 Tagungen im Ausland veranstaltet wurden, vielfach in Kooperation mit den entsprechenden wissenschaftlichen Europavereinigungen anderer Staaten (circa 7 Prozent aller Tagungen des AEI). Als Tagungsorte fallen hier vor allem Wien (5 Tagungen) sowie Tagungsorte mit europäischen Organen auf (Brüssel 4, Luxemburg 3, Straßburg 1). In jüngerer Zeit finden auch verstärkt Konferenzen in englischer Sprache statt.

Thematisch decken die Tagungen fast alle denkbaren Themen der europäischen Integrationsforschung ab. Dabei reicht die Bandbreite von monodisziplinären und fachspezifischen Tagungen hin zu interdisziplinären und die Europäischen Gemeinschaften beziehungsweise die Europäische Union in ihrer Gesamtstruktur umfassenden Konferenzen.

Von Beginn an verband der Arbeitskreis seine jährlichen Gremiensitzungen (Mitgliederversammlung, Vorstands- und Präsidiumssitzung) mit einer wissenschaftlichen interdisziplinären Konferenz: dem Jahreskolloquium. In diesen Kolloquien wurden und werden meist aktuelle Themen der europäischen Integration aus den drei Perspektiven der Politik-, Rechts- und Wirtschaftswissenschaften diskutiert. Häufig wiederkehrende und weit gefasste Themen sind grundlegende Strukturen und Perspektiven der europäischen Integration, die Analyse erfolgter oder kurz bevorstehender Integrationsschritte oder die Rolle der Europäischen Union in der Welt.

In den Jahren 1991 und 1992 hat der AEI vier Tagungen organisiert, die speziell Hochschulangehörigen aus den neuen Bundesländern die Europäische Union aus den Perspektiven der vier Disziplinen Rechts-, Politik-, Wirtschafts- und Geschichtswissenschaften näher bringen sollten.

Tabelle 1: Tagungsorte des AEI nach Häufigkeit ${ }^{3}$

\begin{tabular}{|c|l|c|}
\hline Rang & \multicolumn{1}{|c|}{ Tagungsort } & Anzahl \\
\hline 1 & Bonn & 58 \\
\hline 2 & Berlin & 54 \\
\hline 3 & Hamburg & 23 \\
\hline 4 & Augsburg & 15 \\
\hline 5 & Heidelberg & 11 \\
\hline 6 & Mannheim & 10 \\
\hline 7 & Köln & 7 \\
\hline 8 & Bremen & 6 \\
\hline 9 & Darmstadt & 5 \\
\hline 10 & Wien & 5 \\
\hline
\end{tabular}

Quelle: Eigene Darstellung.

3 Es fehlen Daten zu den Konferenzorten für die Jahre 1977 bis 1981. 
Tabelle 2: Tagungen des AEI nach Teilnehmerzahl Rang 1 bis $10^{4}$

\begin{tabular}{|c|c|c|c|c|}
\hline Rang & Titel der Tagung & Jahr & Ort & $\begin{array}{l}\text { Teilneh- } \\
\text { merzahl }\end{array}$ \\
\hline 1 & $\begin{array}{l}\text { Der Entwurf der Europäischen Verfas- } \\
\text { sung: Die Ergebnisse des Konvents auf } \\
\text { dem Prüfstand }\end{array}$ & 2003 & Berlin & 318 \\
\hline 2 & $\begin{array}{l}\text { Herausforderungen für die Finanzpolitik } \\
\text { in Europa }\end{array}$ & 2007 & Berlin & circa 300 \\
\hline 3 & $\begin{array}{l}\text { Der Vertrag von Nizza - Bewertungen aus } \\
\text { Sicht von Wissenschaft und Politik }\end{array}$ & 2001 & Berlin & 126 \\
\hline 4 & $\begin{array}{l}\text { Legitimations- und Handlungsfähigkeit } \\
\text { der EG nach Maastricht }\end{array}$ & 1991 & Mannheim & 124 \\
\hline 5 & $\begin{array}{l}\text { Die EU und die Regierungskonferenz von } \\
1996 \text { (Jahreskolloquium) }\end{array}$ & 1995 & Bonn & 122 \\
\hline 6 & $\begin{array}{l}\text { Die EU-Strukturpolitik nach 2006: Per- } \\
\text { spektiven für die Reform }\end{array}$ & 2002 & Berlin & 108 \\
\hline 7 & Frauenpolitik in der EU & 1995 & Bonn & 108 \\
\hline 8 & $\begin{array}{l}\text { Der Europäische Verfassungsvertrag - } \\
\text { Grundstein für ein demokratisches und } \\
\text { handlungsfähiges Europa? }\end{array}$ & 2002 & Berlin & 106 \\
\hline 9 & $\begin{array}{l}\text { Auf dem Weg zum Mehrebenenparlamen- } \\
\text { tarismus? }\end{array}$ & 2011 & Tübingen & 99 \\
\hline 10 & $\begin{array}{l}\text { Seerecht und maritime Wirtschaft in der } \\
\text { europäischen Integration }\end{array}$ & 1991 & Hamburg & 97 \\
\hline
\end{tabular}

Quelle: Eigene Darstellung.

Darüber hinaus hat der Arbeitskreis die Wahlen zum Europäischen Parlament durch Tagungen begleitet. 1978 wurden aus Sondermitteln der Europäischen Kommission drei Tagungen veranstaltet, die nicht im offiziellen Tagungsprogramm des AEI figurierten. Eine Tagung widmete sich speziell Studierenden und dem wissenschaftlichen Nachwuchs (,Das Europäische Parlament auf dem Wege zur direkt gewählten Vertretung"), eine wurde für Lehrkräfte und Hochschuldozierende organisiert („Direktwahlen zum Europäischen Parlament“) und eine zielte primär auf JuristInnen und VerfassungshistorikerInnen („Souveränitätsdenken in der EG: Europäische Wahlen und nationale Souveränität, Souveränitätsverständnis und Außenbeziehungen“). Zu den späteren Europawahlen fanden ebenfalls entsprechende Tagungen statt: „Das Europäische Parlament vor der Herausforderung der ersten Wiederwahl“ (Bonn, 1983), „Parlamentarisierung als Beitrag zur Demokratisierung der EG?“ (Bonn, 1990), „Das Europäische Parlament nach der Europawahl 1994“ (Köln, 1994), „Auf dem Weg zu einem europäischen Parteiensystem? - Die Rolle politischer Parteien im EU-System“ (Berlin, 2000), „Das Europäische Parlament nach den Wahlen Funktionen und Aufgabenprofile 2004-2009“ (Berlin, 2004), „National European Debates and the 2009 European Parliament Elections“ (Otzenhausen, 2009) und „Das Europäische Parlament - Funktionswandel und Aufgabenprofile 2014 bis 2019“ (Tutzing, 2014).

4 Es fehlen Daten zur Teilnehmerzahl pro Tagung für die Jahre 1977 bis 1985, 1991 und 1996 bis 1999. 
Der AEI hat neben seinen Konferenzen regelmäßig weitere Vortragsveranstaltungen organisiert. So gab es in den 1970er Jahren einen Gesprächskreis mit US-amerikanischen Gästen aus Wissenschaft und Diplomatie. Die ersten Vortragsabende begannen im Jahr 1973 mit dem damaligen US-Botschafter bei der Europäischen Gemeinschaft, Joseph Greenwald, und dem ehemaligen Vorsitzenden des Council of Economic Advisers, Paul W. McCracken.

Darüber hinaus veranstaltete der AEI in Bonn regelmäßig Veranstaltungen zusammen mit dem Presse- und Informationsamt der Europäischen Kommission. Bei diesen Vortragsabenden referierten oftmals Vertreterinnen und Vertreter der Europäischen Gemeinschaften beziehungsweise der Europäischen Union über aktuelle Themen der europäischen Integration.

\section{Fachzeitschrift und Schriftenreihe mit hoher Reputation}

Der AEI publiziert eine wissenschaftliche Zeitschrift und eine Schriftenreihe im Bereich der europäischen Integrationsforschung.

In der Anfangszeit hatte der AEI keine eigene Fachzeitschrift. 1969 und 1970 erschien allerdings die „Integration - Vierteljahreshefte zur Europaforschung“, die vom Verbindungsbüro der Europäischen Gemeinschaften in Bonn herausgegeben wurde. Bereits damals publizierte die Zeitschrift wissenschaftliche Aufsätze und Tagungsberichte von AEI-Mitgliedern beziehungsweise von AEI-Tagungen. 1971 zog die Redaktion nach Brüssel und die Zeitschrift erschien nur noch in dem Jahr. Beiträge waren in den damaligen vier Amtssprachen der Europäischen Gemeinschaften (Deutsch, Französisch, Italienisch und Niederländisch) sowie in Englisch zugelassen. Schriftleiter der Zeitschrift in den drei Jahren des Erscheinens von 1969 bis 1971 war Hartmut S. Offele.

Erst seit 1981 erschien wieder zweimal jährlich die „Europäische Integration - Mitteilungen des Arbeitskreises“, die zusammen mit dem Verbindungsbüro herausgegeben wurde. In den Mitteilungen wurden überwiegend Tagungsberichte sowie Kurzdarstellungen wissenschaftlicher Arbeiten von AEI-Mitgliedern veröffentlicht. Die Auflage betrug 2.000 Exemplare (1986 bis 1989). Im Jahr 1990 wurden die Mitteilungen aus finanziellen Gründen eingestellt.

Anschließend beteiligte sich der Arbeitskreis an der Zeitschrift integration des Instituts für Europäische Politik (IEP), die unabhängig von den oben erwähnten Zeitschriften bereits seit 1978 vom IEP alleine publiziert wurde. Seit dem 1. Januar 1992 erscheint die integration in der gegenwärtigen Form vierteljährlich als gemeinsame Zeitschrift des IEP und des AEI. Inzwischen wird die integration bei der Nomos Verlagsgesellschaft publiziert und ist die führende interdisziplinäre europawissenschaftliche Zeitschrift in deutscher Sprache.

Im Jahr 1974 wurden zum ersten Mal Tagungsbeiträge gesammelt und als Broschüren publiziert. Im Jahr 1978 erschienen die beiden ersten Bände der Schriftenreihe des AEI bei der Nomos Verlagsgesellschaft mit dem Erscheinen der ersten beiden Bände. Seitdem sind 80 Bände erschienen und die Schriftenreihe hat sich im Bereich der deutschen Europawissenschaften als feste Größe etabliert. Zwei bis drei neue Bände kommen im Schnitt jedes Jahr hinzu. Die meisten der Bücher entstehen als Tagungsbände aus den Konferenzen des AEI und sorgen damit für eine weitere Verbreitung und Bewahrung der Erkenntnisse aus den Tagungen des Arbeitskreises. Die Schriftenreihe dient auch der Sichtbarkeit und wissenschaftlichen Professionalisierung des Arbeitskreises und setzt Orientierungspunkte über die Tagungen hinaus. Die Schriftenreihe hat wissenschaftliche Erkenntnisse zu verschiedenen Themen der europäischen Integrationsforschung gesammelt. $^{5}$

5 Für eine Übersicht über die in der Schriftenreihe erschienen Bänder nach Themen siehe Angang III. 


\section{Über Europa informieren}

Eine wichtige Aufgabe in der Anfangszeit des AEI, die inzwischen nicht mehr wahrgenommen wird, war die Bereitstellung von Informationen über die Tätigkeiten der Europäischen Gemeinschaften und ihrer Aktivitäten. Zu diesem Zweck wurde gleich zu Beginn eine Dokumentationsstelle im Verbindungsbüro der Kommission in enger Zusammenarbeit mit dem AEI aufgebaut. Die Dokumentationsstelle stand Interessierten offen und beantwortete schriftliche Anfragen. Bereits in der Anfangszeit besuchten jeden Monat etwa fünfzig Interessierte die Dokumentationsstelle und darüber hinaus gingen monatlich 180 Anfragen schriftlich ein.

Außerdem bestanden bereits Anfang der 1970er Jahre regelmäßige Kontakte zu über 600 Lehrstühlen und Instituten (Mitte der 1970er Jahre zu über 800 Lehrstühlen und Instituten). Die Dokumentationsstelle verteilte und verschickte die jeweils neu erschienenen Dokumente der Europäischen Gemeinschaften an die deutschen Hochschulen und Forschungseinrichtungen.

Der Arbeitskreis veranstaltete in den ersten Jahren auch Tagungen, die auf die Fortbildung und Vernetzung der Mitarbeiter in den verschiedenen Europäischen Dokumentationszentren an deutschen Hochschulen zielten. Anfang der 1970er Jahre gab es bereits 19 dieser Zentren in Deutschland. Die Dokumentationszentren erhielten alle Dokumente der Europäischen Gemeinschaften. Diese wurden durch die Dokumentationsstellen bereitgestellt und nutzerfreundlich aufbereitet.

Darüber hinaus wurde gemeinsam mit der EG-Kommission eine Liste mit möglichen Dissertationsthemen erstellt und an Hochschulen verteilt, um Anregungen für Forschungsarbeiten zu geben. Des Weiteren organisierte der AEI Informationsbesuche von Hochschulgruppen bei den europäischen Institutionen, wie der Kommission, dem Europäischen Parlament oder dem Europäischen Gerichtshof.

Neben der Bereitstellung von Informationen bestand eine weitere Aufgabe in der Anfangszeit des Arbeitskreises in der Dokumentation der Lehre über die europäische Integration an deutschen Hochschulen. Im Wintersemester 1971/1972 und im Sommersemester 1972 wurden 140 Vorlesungen, Übungen und Seminare zu Themen der europäischen Integration an deutschen Hochschulen gezählt, davon 63 rechtswissenschaftliche, 57 wirtschaftswissenschaftliche, 16 politikwissenschaftliche sowie vier geschichtswissenschaftliche Veranstaltungen. Dies bewegte sich in etwa auf dem Niveau der Lehre in Frankreich zu Themen der europäischen Integration und stellte mehr als eine Verdoppelung im Vergleich zum Zeitraum 1966/1967 dar.

Seit 1990/1991 nimmt der Arbeitskreis die Aufgabe der Dokumentation und Information der Arbeit der Europäischen Gemeinschaften nicht mehr wahr, da die Kommission ihre Arbeit auf eine neue Grundlage stellte und in diesem Bereich erhebliche Kürzungen vornahm. Die Arbeit der Dokumentation und Information über die Europäischen Gemeinschaften war vor allem in einer Zeit nützlich, in der noch nicht alle Dokumente online verfügbar waren.

\section{Vielfältige Partner}

Der Arbeitskreis hat vielfältige Partner. Hiervon ist die Europäische Kommission der älteste und engste Kooperationspartner des Arbeitskreises. Das Verbindungsbüro der Kommission hat bereits bei der Gründung des AEI eine maßgebliche Rolle gespielt. In der Anfangszeit hat der AEI über die Tätigkeit der Europäischen Gemeinschaften informiert, Räumlichkeiten im Verbindungsbüro genutzt und das Personal sowie der größte Teil der Ausgaben für Tagungen wurden direkt durch die Kommission bezahlt. Der Geschäftsführer übernahm in Personalunion Aufgaben für das Verbindungsbüro als sogenannter, animateur universitaire‘ und pflegte in dieser Funktion unter anderem die Kontakte zu den europäischen Dokumentationszentren in Deutschland, organisierte Gruppenbesuche von Hochschulangehörigen bei den EG-Institutionen und repräsentierte das Verbindungsbüro im Hochschulbereich. Im ersten Jahrzehnt war zudem der Leiter des Ver- 
bindungsbüros der Europäischen Gemeinschaften Mitglied im Vorstand des AEI und nahm auch dadurch Einfluss auf die inhaltliche Ausrichtung der Aktivitäten des Arbeitskreises.

Von Anfang an haben beide Seiten aber auch Wert auf die gegenseitige Unabhängigkeit gelegt und so wurde beispielsweise schon 1976 eine räumliche Trennung zwischen dem AEI und dem Verbindungsbüro der Kommission umgesetzt. Allerdings blieb der AEI noch bis 1991 im gleichen Haus wie das Verbindungsbüro. Diese erste Phase der vergleichsweise engen Anbindung des Arbeitskreises an die Europäische Kommission dauerte bis Anfang der 1990er Jahre an. In diesen Jahren wurde die Informations- und Dokumentationstätigkeit des Arbeitskreises für die Kommission eingestellt und es wurden neue Räumlichkeiten in der Bachstraße in Bonn bezogen, in denen das IEP und die Europa-Union bereits ihren Sitz hatten. Das IEP und der AEI gaben seitdem gemeinsam die Zeitschrift integration heraus und der AEI verzichtete fortan auf die Mitarbeit an Publikationen der Europäischen Gemeinschaften beziehungsweise der Europäischen Union. Bereits in dieser Zeit wurde auch der Zuschuss der Kommission für die Geschäftsstelle des AEI reduziert. Seit dieser Zeit werden die laufenden Kosten des Arbeitskreises (Personal, Verwaltung) zum wesentlichen Teil durch die Beiträge seiner Mitglieder gedeckt.

Eine zweite Zäsur fand Anfang der 2000er Jahre statt, als die Kommission die direkte Finanzierung der Geschäftsstelle des AEI vollständig beendete. Die Tagungen finanziert der AEI noch immer zu einem wichtigen Teil (circa 40 Prozent der Kosten) aus Projektgeldern der Europäischen Kommission. Allerdings muss sich der Arbeitskreis dabei in der starken und zunehmenden Konkurrenz mit anderen Antragstellern behaupten und jedes Jahr erneut mit der Qualität seiner Tagungsprojekte überzeugen.

Der größere Teil der Konferenzfinanzierung stammt von den vielen anderen Partnern des Arbeitskreises. Der AEI organisiert seine Konferenzen in der Regel mit Kooperationspartnern. Entsprechend hat der Arbeitskreis im Laufe seiner Geschichte mit Hunderten Hochschulen, Forschungseinrichtungen, Forschungsfördereinrichtungen, wie der Deutschen Forschungsgemeinschaft, Akademien der politischen Bildung, Stiftungen, Europavereinigungen, Landes- und Bundesministerien, Europazentren, wissenschaftlichen Vereinigungen, Landesvertretungen bei der Europäischen Union, Botschaften, Bundes- und Landeszentralen für politische Bildung sowie der Deutschen Bundesbank zusammengearbeitet. Vereinzelt haben auch Unternehmen oder Unternehmensstiftungen Tagungen des AEI gefördert.

Seit 1993 ist der Arbeitskreis zudem Mitglied des Netzwerkes Europäische Bewegung Deutschland e.V., dem Verband der deutschen europapolitischen Akteure.

Neben Kooperationspartnern aus Deutschland ist der Arbeitskreis auch international eng vernetzt. Der Arbeitskreis ist Gründungsmitglied des Europäischen Komitees für die Zusammenarbeit der nationalen Vereinigungen von Hochschullehrern und Wissenschaftlern, die sich mit der europäischen Integration befassen. Die Gründung erfolgte auf einem Treffen am 16. November 1987 am Europäischen Hochschulinstitut in Florenz. Die anderen Gründungsmitglieder neben dem AEI sind die Vereinigungen aus Dänemark (Dansk Selskab for Europaforskning), Frankreich (Commission pour l'Étude des Communautés Européennes), Großbritannien (University Association for Contemporary European Studies, UACES), Irland (Irish Association for Contemporary European Studies) und der Niederlande (Interdisciplinaire Studiegroep Europese Integratie). Allerdings gab es bereits vorher einen regelmäßigen Austausch und bereits 1976 einen Vorschlag der britischen Vereinigung UACES, ein internationales Büro zu gründen. Der Vorschlag wurde allerdings damals noch von den anderen nationalen Vereinigungen abgelehnt.

Schließlich formierte sich das genannte europäische Komitee als European Community Studies Association (ECSA) als eingetragener Verein in Belgien. Es firmierte zuerst als ECSA Europe und später als ECSA World, als andere kontinentale Verbände, wie ECSA Asia, sich gründeten. Inzwischen sind über sechzig nationale wissenschaftliche Europavereinigungen Mitglied. Von 
1998 bis 2000 war der damalige Vorstandsvorsitzende des AEI, Rudolf Hrbek, Präsident von ECSA World.

Eines der ersten Projekte der neu gegründeten ECSA war die Herausgabe eines englisch- und französischsprachigen Verzeichnisses der im Bereich der europäischen Integration tätigen Personen in neun Mitgliedstaaten (Belgien, Dänemark, Deutschland, Frankreich, Großbritannien, Irland, Italien, Niederlande und Spanien). Dieses Verzeichnis erschien in fünf Auflagen. Zuerst erschien ein Verzeichnis „Who is Who in European Integration“ der Experten aus EG- beziehungsweise EU-Mitgliedstaaten in drei Auflagen (1989, 1991 und 1995). Anschließend wurde ein Verzeichnis von Experten aus Nicht-EU-Staaten erstellt und 1996 veröffentlicht: „Who is Who in European Integration Studies (in Non-EU-States)“. Im Jahr 2000 wurde schließlich ein globales Verzeichnis von EU-Experten publiziert: „Who is Who in European Integration Studies“. Alle fünf Auflagen wurden unter Federführung des AEI erstellt. Aufgrund der leichteren Suchmöglichkeit nach EU-Experten durch das Internet wurde ein solches Verzeichnis nach 2000 nicht wieder aufgelegt. Die Geschäftsstelle des AEI hat in den ersten Jahren die ECSA insgesamt organisatorisch unterstützt.

Der AEI hat mehrfach Tagungen mit anderen ECSA-Vereinigungen zusammen veranstaltet, beispielsweise mit ECSA Turkey in Istanbul oder mit ECSA World in Berlin (beide in 2009). Eine besonders enge Zusammenarbeit besteht mit den beiden anderen deutschsprachigen ECSAVereinigungen, ECSA Austria und ECSA Suisse. Seit 1997 finden, abwechselnd in Deutschland, Österreich oder der Schweiz und in regelmäßigen Abständen, gemeinsame Tagungen statt. Die inzwischen zwölfte dieser Dreiländertagungen ist für Oktober 2014 in Lausanne (Schweiz) geplant.

\section{Der AEI in Bonn und Berlin}

Der AEI war in den ersten beiden Jahrzehnten in den Räumen des deutschen Verbindungsbüros der Europäischen Gemeinschaften in Bonn angesiedelt. 1991 erfolgte der Umzug in das Institut für Europäische Politik in der Bachstraße 32 und im Jahr 2000 in das Zentrum für Europäische Integrationsforschung (beide in Bonn). Im Jahr 2003 folgte schließlich der Umzug nach Berlin in die Stiftung Wissenschaft und Politik.

\section{Netzwerk aus Wissenschaft und Praxis}

Der Arbeitskreis verfügt über aktuell rund vierhundert Mitglieder. Sie sind überwiegend Hochschullehrerinnen und Hochschullehrer und wissenschaftliche Mitarbeiterinnen und Mitarbeiter vor allem aus den drei Disziplinen der Politik-, Rechts- und Wirtschaftswissenschaften. Darüber hinaus sind ebenfalls viele Mitglieder Personen, die im Bereich der europäischen Integration tätig sind: auf nationaler Ebene, etwa aus der Administration von Bund und Ländern, sowie aus den europäischen Institutionen.

Die Zahl der Mitglieder betrug bei der Gründung 24 und wird danach zum zweiten Mal im Tätigkeitsbericht 1972/1973 dokumentiert und mit 95 angegeben. Die Zahl wuchs auf 227 (1980), 315 (1985), 330 (1990) und 390 (1995). Seitdem hat sich die Mitgliederzahl mit geringfügigen Schwankungen bei etwa vierhundert Mitgliedern eingependelt. 


\section{Anhang I}

Die Präsidenten des AEI

Prof. Dr. Walter Hallstein

(1969-1975)

Prof. Dr. Alfred Müller-Armack

(1975-1978)

Prof. Dr. Bodo Börner

(1978-1990)

Dr. Karl-Heinz Narjes

(1990-1999)

Prof. Dr. Thomas Oppermann

(1999-2002)

Prof. Dr. Rudolf Hrbek

(seit 2002)

Die Vorstandsvorsitzenden des AEI

Prof. Dr. Hermann Priebe

(1969-1975)

Prof. Dr. Ronald Clapham

(1975-1981)

Prof. Dr. Beate Kohler-Koch

(1981-1985)

Prof. Dr. Manfred Zuleeg

(1985-1988)

Dr. Fritz Franzmeyer

(1988-1989)

Prof. Dr. Beate Kohler-Koch

(1989-1990)

Prof. Dr. Rudolf Hrbek

(1990-2002)

Prof. Dr. Dr. h.c. mult. Peter-Christian Müller-Graff (seit 2002)

Die Geschäftsführer des AEI

Hans-Joachim vom Berge

(1969-1975)

Dr. Holger Mirek

(1976-1980)

Horst Zeller

(1980-1989)

Armin Czysz

(1989-1990)

Meike Leube

(1990-2002)

Matthias Heddenhausen

(2002-2008)

Katharina Joho

(2008-2009)

Dr. Frédéric Krumbein

(seit 2009) 


\section{Anhang II: Die Konferenzen des AEI*}

2013

1) Grenzen der Integration - Europas strategische Ansätze für die Nachbarregionen (Berlin, 44, Band 79)

2) Police Cooperation in the EU under the Treaty of Lisbon-Opportunities and Limitations (Berlin, 77)

3) National Parliaments in the EU - The Performance of Multilevel Democracy in Europe (Berlin, 69)

4) The EU between an "ever closer union" and inalienable policy domains of members states (Otzenhausen, 45, Band 80)

5) Fiscal Performance - The Role of Institutions and Politicians (Mannheim, 47)

6) Jahreskolloquium: EU und USA - Europas transatlantische Aufgaben (Heidelberg, 72)

\section{2}

1) Polen als Motor des europäischen Integrationsprozesses - Ergebnisse der polnischen Ratspräsidentschaft (Chemnitz, 66, Band 77)

2) Zug- und Gegenkräfte im europäischen Integrationsprozess - Gefahr eines wachsenden Ungleichgewichts? (Genshagen, 30, Band 78)

3) Innen- und Justizpolitik in der gemeinsamen $\mathrm{Au}-$ ßen- und Sicherheitspolitik (Berlin, 58)

4) Kommunikation im Europa der Krise (Berlin, 36)

5) International Governance for Renewable Energy and the Role of Emerging Powers (Berlin, 50)

6) Social Media in der europapolitischen Kommunikation (Stuttgart, 83)

7) Auf dem Weg zur europäischen Sportpolitik (Köln, 66)

8) Die dritte Gewalt in Europa (Kiew, ca. 35)

9) Verbesserungen im Zivilprozess in Europa? (Potsdam, ca. 60)

10) 11. Dreiländertagung von ECSA Austria, ECSA Suisse und des AEI und Jahreskolloquium: Projekt Europa - Zwischen Solidarität und Desintegration (Darmstadt, 54)

\section{1}

1) Die Donaustrategie der Europäischen Union (Berlin, 50)

2) First German ECSA Young Researcher Conference "Europe's Post-Crisis Stability"(Berlin, 24)

3) Post-Crisis Fiscal Consolidation Strategies for Europe (Freiburg, 32)

4) Europäisierte Regulierungsstrukturen und -netzwerke als Basis einer künftigen Infrastrukturvorsorge (Leipzig, 48, Band 74)
5) Auf dem Weg zum Mehrebenenparlamentarismus? (Tübingen, 99, Band 75)

6) Sicherheitspolitische Utopie oder energiepolitische Vision? - Deutschland, Frankreich und die Beziehungen der EU zu den islamischen Mittelmeerländern (Kassel, 65)

7) Jahreskolloquium: Marginalisierung Europas? (Heidelberg, 54)

\section{0}

1) Die europapolitischen Perspektiven des Balkans (Jena, 44)

2) Dreiländertagung: Kleine Staaten innerhalb und außerhalb der EU (Wien, ca. 30)

3) Wechselwirkungen von Migration und Integration im europäischen Mehrebenensystem (Bremen, 67)

4) Das „soft law“ der Europäischen Organisationen (Straßburg, ca. 75)

5) The EU in International Fora - Lessons for the Union's External Representation after Lisbon (Berlin, 38, Band 72)

6) Die Vertragsbeziehungen der EU mit europäischen Nachbarstaaten - Das Ukraine-Abkommen (Kiew, ca. 40)

7) Die gemeinsame Handelspolitik der EU „nach Lissabon" (Berlin, 65, Band 73)

8) Konvergenz der Unternehmensrechte (Nancy, ca. 60)

9) Jahreskolloquium: Die Lissabonner Reformen im ersten Test von Praxis und Krise: Konsequenzen und Kontroversen (Bochum, ca. 45)

\section{9}

1) Measuring the Europeanization of Public Policies Beyond the $80 \%$-Myth (Berlin, 50)

2) Rechtsstaat, Freiheit und Sicherheit in Europa (Budapest, ca. 50)

3) Die europäischen Perspektiven der Türkei (Istanbul, 60, Band 76)

4) Die Europäische Union 2020: Entwicklungsperspektiven der Integration (Bonn, 36)

5) The Economics of Charitable Giving (Mannheim, 33)

6) National European Debates and the 2009 European Parliament Elections (Otzenhausen, 22, Band 71)

7) Differenzierte Integration im Privatrecht (Heidelberg, ca. 60)

* Soweit bekannt finden sich in den Klammern der Tagungsort und die Zahl der Teilnehmer. Sofern es zur Tagung einen Tagungsband gab, findet sich in Klammern ebenfalls die Nummer des Bandes in der Schriftenreihe des AEI bei der Nomos Verlagsgesellschaft. 
8) Jahreskolloquium: Krisen als Chancen der Europäischen Union (Berlin, 43)

9) „Horizont 2020-2030“ - Herausforderungen des Mehrebenensystems an die EU-Reflexionsgruppe (Stuttgart, 67, Band 69)

\section{8}

1) Kommunale Aufgaben im Spannungsfeld zwischen Gemeinwohl und Binnenmarkt (Neuhausen/Stuttgart, 67, Band 68)

2) The European Antitrust-Rules - The 2003 Reform and Its Consequences (Nürnberg, 19)

3) Ökonomik, Politische Ökonomie und Psychologie staatlicher Reformen in Europa (Freiburg, 37)

4) Die Europäisierung der Kontrolle: Deutschland und die Schaffung eines Raums der Freiheit, der Sicherheit und des Rechts (Berlin, 45)

5) Europäisches Gesellschaftsrecht auf neuen Wegen (Heidelberg, 66, Band 67)

6) Europe in Transition - Demographic Change and Sustainable Development (Essen, 84)

7) 9. Dreiländertagung: Europa und die Demokratie (Luzern, 28)

8) Jahreskolloquium: Die EU als Akteur der Weltordnungspolitik (Heidelberg, 63)

9) The Euro at Ten: Governing the Eurozone in a Globalised World Economy (Berlin, 44)

\section{7}

1) Herausforderungen für die Finanzpolitik in Europa (Berlin, ca. 300)

2) Die Außenpolitik der Europäischen Union: Zwischen interregionalem Dialog und strategischer Partnerschaft (Berlin, 61)

3) Die Europäische Gesundheitspolitik - effektiv und bürgernah auch mit wenig Kompetenzen (Weimar, 16)

4) Nachbarschaftspolitik der EU gegenüber beitrittswilligen osteuropäischen Demokratien (Krakau, 80 , Band 65)

5) The Eurozone under stretch? (Berlin, 70)

6) 50 Jahre Römische Verträge: eine rückblickende Bewertung des Integrationsprozesses und Ausblick auf die Zukunft Europas (Berlin, 70)

7) Rechtstaatlichkeit und Gemeinschaftsgerichtsbarkeit (Heidelberg, 76)

8) Jahreskolloquium: Superstaat EU? (Berlin, 42)

9) Europe and New Leading Powers: Towards Partnership in Strategic Policy Areas (Berlin, 31, Band 66)

10) Juristenausbildung in Europa zwischen Tradition und Reform (Trier, 70)

\section{6}

1) Economic Growth in Europe (Mannheim, 35)

2) Sharing the Growing Economic Burden of World Order - How to Strengthen the Economic Base of Foreign and Security Policy in the Atlantic Alliance (Berlin, 46)

3) Nachbarschaften innerhalb der Europäischen Union (Budapest, 80, Band 62)

4) Draußen vor der Tür? - Konzeptionelle Herausforderungen für die EU-Politik gegenüber den neuen Nachbarn (Berlin, 70)

5) Management Reforms in International and Supranational Organisations (Konstanz, 62)

6) 8. Dreiländer-Tagung von AEI, ECSA Austria und ECSA Suisse: Die Schweiz und Europa (Heidelberg, 46, Band 61)

7) Migrationspolitik nach dem Amsterdamer Vertrag - Die Europäische Union zwischen völkerrechtlicher Verpflichtung und mitgliedstaatlichen Interessen (Frankfurt, 36, Band 63)

8) Medien und transnationale Kommunikation in der Europäischen Gemeinschaft (Mainz, 37, Band 59)

9) „Bittere Erfahrungen“ der Vergangenheit und der Prozess der Konstitutionalisierung Europas (Berlin, 76)

10) Jahreskolloquium: Was Europa zusammenhält. In Vielfalt geeint? (Berlin, 50, Band 64)

\section{5}

1) Prioritätenwechsel in der EU-Ausgabenpolitik? Agenda 2007 im Zeichen der Lissabon-Agenda (Otzenhausen, 23)

2) Verfassungsentwicklung zwischen den europäischen Gipfeln - Chancen und Grenzen des intraund interinstitutionellen ,soft law" in der EU (Berlin, 19)

3) Europapolitik im System des Rates der Europäischen Union (Berlin, 39)

4) Die EG in der internationalen Umweltpolitik (Würzburg, 88)

5) Die Außenwirtschaftspolitik der Europäischen Union nach dem Verfassungsvertrag (München, 44, Band 55)

6) The Future European Party System (Tübingen, 30)

7) 7. Dreiländertagung von ECSA Austria, ECSA Suisse und dem AEI: Kerneuropa - Differenzierte Integration als Konzept für die erweiterte EU oder Grundlage für ihre Spaltung? (Wien, 90)

8) Jahreskolloquium: Deutschlands Rolle in der Europäischen Union (Berlin, 80, Band 60)

9) The EU as an External Democracy Promoter: East and Central Europe, the Mediterranean, Asia, Latin America, the Caribbean, Africa and Pacific (ACP) in Comparison (Darmstadt, 38, Band 58) 


\section{4}

1) Die EU-Strukturpolitik nach 2006: Perspektiven für die Reform (Berlin, 108, Band 53)

2) Effizienter und demokratischer? - Die Verfassung der EU nach der Regierungskonferenz (Bremen, 33)

3) New stimulus or integration backlash? EU enlargement and transatlantic relations (Berlin, 24)

4) Das Europäische Parlament nach den Wahlen Funktionen und Aufgabenprofile 2004-2009 (Berlin, 78, Band 54)

5) Der Europäische Verfassungsvertrag - Grundstein für ein demokratisches und handlungsfähiges Europa? (Berlin, 106)

6) 6. Dreiländertagung von ECSA Austria, ECSA Suisse und dem AEI und Jahreskolloquium: Die Rolle der erweiterten Europäischen Union in der Welt (Berlin, 81, Band 57)

7) The European Union's Policy of Gender Equality Implications of deeper Integration and further Enlargement (Hamburg, 74, Band 52)

1) Democracy and Accountability in the Enlarged European Union (Berlin, 54)

2) Europäisierung der Zivilgesellschaften oder Europäische Zivilgesellschaft: Konzepte, Akteure, Strategien (Mannheim, 36)

3) Raum der Sicherheit, der Freiheit und des Rechts (Heidelberg, 41, Band 51)

4) Regulation and Supervision of Financial Markets and Institutions in the EU (Mannheim, 67)

5) Der Entwurf der Europäischen Verfassung: Die Ergebnisse des Konvents auf dem Prüfstand (Berlin, 318)

6) Jahreskolloquium: Die Wirtschaftsverfassung der Europäischen Union: Gegenwart und Perspektive des Verfassungsentwurfs (Berlin, 69)

7) 5. Dreiländertagung von ECSA Austria, ECSA Suisse und dem AEI: The Future of the World Trading System after the Failure of Cancún (Wien, 81)

8) Europa auf dem Wege zum wettbewerbsfähigsten und dynamischsten Wirtschaftsraum der Welt? Eine Zwischenbilanz der Lissabon-Strategie (Hamburg, 30)

2002

1) Der Gipfel von Laeken und der Post-Nizza Prozess - Analyse und Bewertung aus Sicht von Wissenschaft und Politik (Berlin, 80)

2) Spezifika einer Südost-Erweiterung der EU: Die Türkei und die EU-Türkei-Beziehungen (Wildbad Kreuth, 59, Band 49)

3) Öffentliche Daseinsvorsorge: Nationale Einrichtungen und Europäische Integration (Bonn, 38)
4) Konvergenz und Divergenz in der EU: Empirische Befunde und Wirtschaftspolitische Implikationen (Hamburg, 48)

5) 4. Drei-Länder-Tagung von ECSA Austria, ECSA Suisse und des AEI: Perspektiven der Außenwirtschaftspolitik in der EU und der WTO (Bern, 47)

6) The Mediterranean in the New Evolving International Order - Domestic, Regional and International Interests (Mainz, 21)

7) Jahreskolloquium: Folgeprobleme der EU-Osterweiterung (Bonn, 71)

\section{1}

1) Der Vertrag von Nizza - Bewertungen aus Sicht von Wissenschaft und Politik (Berlin, 126)

2) Towards an Assertive Europe - EU's international Presence (Mannheim, 50)

3) Der unvollendete Binnenmarkt (Hamburg, 45)

4) Weissbuch „Governance“ der Europäischen Kommission (Berlin, 50)

5) Jahreskolloquium: Die EU als internationaler Akteur (Bonn, 66)

6) Kirchen und Religionsgemeinschaften in der Europäischen Union (Heidelberg, 77, Band 50)

\section{0}

1) Die Europäische Kommission nach Amsterdam: zwischen Mega-Bürokratie und politischer Steuerungsinstanz (Bonn, 46)

2) Die Wirtschafts- und Währungsunion: Eine Zwischenbilanz (Hamburg, 58)

3) Auf dem Weg zu einem europäischen Parteiensystem? - Die Rolle politischer Parteien im EU-System (Berlin, 39)

4) EU-Osterweiterung und Finanzmärkte (Mannheim, 45)

5) Jahreskolloquium: Die EU vor der Erweiterung - Reformerfordernisse im Lichte der Regierungskonferenz 2000 (Bonn, 82)

6) Die Beziehungen zwischen der EU und den MERCOSUR-Staaten - Stand und Perspektiven (Tutzing, 30, Band 48)

\section{9}

\section{(370 Teilnehmer insgesamt)}

1) Die EG in der Welthandelsorganisation - Europa und die Globalisierung (Heidelberg, Band 47)

2) Studying Europe: Challenges and Prospects for European Studies (Bremen, Band 45)

3) Arbeitsmarkt- und Beschäftigungspolitik in der Europäischen Union: Nationale und europäische Perspektiven (Würzburg, Band 46)

4) Europa nach den Beschlüssen der „Agenda 2000“ (Hamburg)

5) Stand und Entwicklung theoretischer Konzepte zum europäischen Integrationsprozess (Essen) 
6) Jahreskolloquium und 3. Dreiländertagung von ECSA Austria, ECSA Suisse und dem AEI: Institutionen und Gemeinschaftspolitiken der EU vor der Erweiterung (Bonn)

\section{8}

\section{(655 Teilnehmer insgesamt)}

1) Rolle der Regionen in der neuen EU (Bremen)

2) Nach den Beschlüssen von Brüssel: Die Wirtschafts- und Währungsunion vor dem Start (Hamburg)

3) Emile Noël: Rolle und Persönlichkeit (Bonn)

4) Mittelmeerpolitik der EU (Tutzing, Band 44)

5) 2. Dreiländertagung von ECSA Austria, ECSA Suisse und dem AEI: Außenwirtschaft und Außenpolitik der EU (Wien)

6) Jahreskolloquium: Die EU vor den Herausforderungen der Globalisierung (Bonn)

7) Deutschland und die EU: Die deutsche Präsidentschaft des Rates der EU (Bonn)

\section{7}

\section{(661 Teilnehmer insgesamt)}

1) Finanzverfassung und Strukturpolitik der EU (Bremen, Band 42)

2) Die Gemeinsame Außen- und Sicherheitspolitik der Europäischen Union: Leistungskraft, Defizite, Reformvorhaben (Bonn)

3) 40 Jahre Römische Verträge: Der deutsche Beitrag (Baden-Baden)

4) Der Amsterdamer Vertrag unter der Lupe: Politik und Wissenschaft im Diskurs (Bonn)

5) 1. Dreiländertagung von ECSA Austria, ECSA Suisse und dem AEI: Eine Standortbestimmung nach Amsterdam: Die Europäische Umwelt-, Verkehrs- und Justizpolitik im Dreiländer-Vergleich (Basel)

6) Jahreskolloquium: Europa 2000: Die Herausforderungen der EU nach der Regierungskonferenz (Bonn)

7) Regierungskonferenz 1996 und Osterweiterung der EU unter besonderer Berücksichtigung der Bereiche Inneres und Justiz (Dritte Säule) (Erfurt)

\section{6}

\section{(532 Teilnehmer insgesamt)}

1) Die institutionellen Neuerungen des Maastrichter Vertrags: Zwischenbilanz, Reformbedarf und die Vorschläge der Reflexionsgruppe (Bonn)

2) Europäisierung von Hochschule, Studium und Forschung (Bonn)

3) Die soziale Dimension der Europäischen Union: Entwicklungen, Herausforderungen und Perspektiven (Tutzing, Band 40)
4) Bestandsaufnahme und Perspektiven der Heranführung der Mittel- und Osteuropäischen Länder an die EU am Beispiel Polens (Frankfurt/Oder, Slubice)

5) Jahreskolloquium: Deutschland in Europa (Bonn)

6) Gemeinsames Verfassungsrecht in der Europäischen Union (Heidelberg, Band 43)

7) Die Beziehungen zwischen der EU und Asien unter besonderer Berücksichtigung von ASEAN (Bonn)

\section{5}

1) Frauenpolitik in der EU (Bonn, 108, Band 37)

2) Die Europäische Zusammenarbeit in den Bereichen Justiz und Inneres (JIZ): der Dritte Pfeiler der EU (Bonn, 60, Band 38)

3) Polen und die Osterweiterung der EU (Berlin, 75)

4) Transeuropäische Netze (Tutzing, 35, Band 39)

5) Jahreskolloquium: Die EU und die Regierungskonferenz 1996 (Bonn, 122, Band 41)

6) Überlegungen zur wissenschaftlichen Analyse und Vermittlung von Beiträgen zur Regierungskonferenz 1996 (Bonn, 24)

\section{4}

1) Die EU und die Visegrad-Staaten: Bilanz und Optionen der Assoziierungspolitik (Bonn, 74)

2) Die europapolitische Strategiedebatte (Bonn, 56)

3) Schwerpunkte der Uruguay-Runde und mögliche Auswirkungen auf eine künftige Weltwirtschaftsordnung (Augsburg, 36)

4) Das Europäische Parlament nach der Europawahl 1994 (Köln, 53)

5) Subsidiaritätsprinzip und europäische Integration: Auswirkungen auf einzelne Politikfelder (Tübingen, 55, Band 36)

6) Jahreskolloquium: EU und Standortqualität: Zur europäischen Debatte zum Weißbuch (Bonn, 71)

7) Die Vierte Erweiterung der Europäischen Union: Von der EFTA über den EWR zur Mitgliedschaft (Bonn, 80)

1993

1) Von der Europäischen Politischen Zusammenarbeit (EPZ) zur Gemeinsamen Außen- und Sicherheitspolitik (GASP) - Kohärenz und Leistungsfähigkeit der EG/Zwölf (Bonn, 58)

2) Kommunale Ebene und EG-Integration (Bocholt, 41)

3) Konvergenz als zentrale Aufgabe der Wirtschaftspolitik im Zeichen von Maastricht (Berlin, 52)

4) Ausgewählte Fragen der Außenbeziehungen der EG im Lichte wirtschaftswissenschaftlicher Analyse (Jena, 39) 
5) The Third Pillar of Maastricht - Cooperation in the Fields of Justice and Home Affairs as a New Dynamic Area of European Politics (Brügge, 20)

6) Jahreskolloquium: Bürger und Europa (Bonn, 67, Band 35)

7) Seerecht und maritime Wirtschaft in der europäischen Integration (Hamburg, 97)

8) Legitimations- und Handlungsfähigkeit der EG nach Maastricht (Mannheim, 124)

\section{2}

1) Verlauf und Ergebnis der Regierungskonferenzen (Bonn, 87)

2) Gemeinschaftsrecht und Privatrecht (Trier, 96, Band 33)

3) Das EG-Finanzsystem - Gegenwärtiger Stand nach Maastricht und künftige Entwicklungschancen (Bonn, 55)

4) Konzeption und Entwicklung der EG im Lichte politikwissenschaftlicher Analyse (Berlin, 44)

5) Konzeption und Entwicklung der EG im Lichte rechtswissenschaftlicher Analyse (Berlin, 48)

6) Jahreskolloquium: Der Maastrichter Vertrag über die EU in der wissenschaftlichen Kontroverse (Bonn, 91, Band 34)

7) Die Reform der Agrarpolitik - Auswirkungen auf die politische Integration und die Außenbeziehungen der Gemeinschaft (Augsburg, 37)

1) Perspektiven der Gemeinschaftsentwicklung: Die Regierungskonferenzen zur Wirtschafts- und Währungsunion und zur Politischen Union (Bonn)

2) Vertiefung versus Erweiterung der Gemeinschaft (Brügge)

3) Konzeption und Entwicklung der EG im Lichte wirtschaftswissenschaftlicher Analyse (Berlin)

4) Jahreskolloquium: Die Entwicklung der EG zur Politischen Union und zur Wirtschafts- und Währungsunion unter der Sonde der Wissenschaft (Bonn, Band 32)

5) Die Beziehungen zwischen der EG und ostmitteleuropäischen Staaten: Möglichkeiten und Grenzen von Europa-Abkommen (Augsburg)

6) Entstehung und Entwicklung der EG im Lichte zeitgeschichtlicher Analyse (Bamberg)

\section{0}

1) Parlamentarisierung als Beitrag zur Demokratisierung der EG? (Bonn, 50)

2) Der Schutz des geistigen Eigentums im Europäischen Binnenmarkt (Bielefeld, 46, Band 29)

3) Deutsch-deutsche Wirtschafts-,Währungs- und Sozialunion im Rahmen der EG (Dresden, 49, Band 30)
4) Beziehungen der EG zu Ländern der Dritten Welt mit neuen Akzenten? (Hamburg, 32)

5) Jahreskolloquium: EU und Drittlandsbeziehungen nach 1992 (Bonn, 82, Band 31)

6) Die Auswirkungen des Binnenmarktes auf die Entwicklung der Regionen in der Europäischen Gemeinschaft (Berlin, 70)

\section{9}

1) Freiheit des Dienstleistungsverkehrs und Vollendung des Binnenmarktes (Glashütten/Oberems, 52)

2) Die Süderweiterung der Gemeinschaft - eine kritische Bestandsaufnahme (Osnabrück, 39)

3) Die Wasserwirtschaft (in) der EG (Berlin, 55)

4) Die EG im Spannungsfeld von zwischenstaatlicher Kooperation und Integration (Hamburg, 45)

5) Steuerharmonisierung und Binnenmarkt (Lambrecht, 37)

6) Europa 1992 - Konzeptionen, Strategien und Außenwirkungen (Konstanz)

7) Die soziale Dimension der Vollendung des Europäischen Binnenmarktes (Hamburg, 48, Band 27)

8) Technische Regeln (Normen und Standards) - Bedeutung für die Vollendung des Binnenmarktes (Bonn, 39, Band 28)

9) Jahreskolloquium: Vertikale Machtverteilung in der Europäischen Gemeinschaft (Augsburg, 35)

\section{8}

1) Beziehungen zwischen der EG und dem Rat für gegenseitige Wirtschaftshilfe in den 90er Jahren: Perspektiven und Probleme (Augsburg, 45)

2) Das Gesundheitswesen in der EG (Hamburg, 42)

3) Integrationsprozesse in der EG und in den USA (Bonn, 51)

4) Bildungs- und Kulturpolitik in der EG (Augsburg, 42)

5) Jahreskolloquium: Wechselwirkungen zwischen der Gemeinsamen Agrarpolitik und anderen Politikbereichen (Bonn, 48)

6) Das EG-Finanzsystem - Probleme und Lösungsmöglichkeiten (Lohmar, 36)

\section{7}

1) Europäisches Umweltrecht und europäische Umweltpolitik (Osnabrück, 74)

2) Der Beitrag der EG zur Gleichstellung von Frau und Mann (Oldenburg, 64)

3) Europäische Aspekte der Ausländerpolitik (Hamburg, 49, Band 26)

4) Kleine und mittlere Unternehmen in der EG (Augsburg, 48)

5) Der Beitrag der EG zur Neuordnung des Weltwährungssystems (Bonn, 51) 
6) Alternativen der Energiepolitik in der EG (Darmstadt, 36)

7) Jahreskolloquium: Die Einheitliche Europäische Akte

\section{6}

1) Die Beziehungen zwischen ASEAN und der EG (Passau, 61)

2) Die Beziehungen zwischen der Türkei und der EG (Augsburg, 45)

3) Europäisierung der Gewerkschaften in der Krise: Die Auswirkungen der EG-Integration auf Organisation und Politik der Gewerkschaften (Hamburg, 31)

4) Vollendung des Binnenmarktes der EG (Hamburg, 52)

5) Nationale Ordnung der Kommunikationsstrukturen - ein Integrationshemmnis? (Feldkirchen-Westerham, 40)

6) Der Beitrag der EG zur Bekämpfung des Hungers in der Welt (Berlin, 48)

7) Jahreskolloquium: Die Rolle des Europäischen Parlaments - Bestandsaufnahme und Ausblick (Luxemburg, 96)

\section{5}

1) Die Luftverkehrspolitik in der EG (Seeheim)

2) Neue Finanzierungsformen der Gemeinschaft (Lambrecht)

3) Ökonomie und Ökologie der Gemeinschaftsmeere (Hamburg)

4) Sicherheitspolitische Zusammenarbeit und Kooperation der Rüstungswirtschaft in Westeuropa (Ludwigshafen, Band 25)

5) Technologiepolitik als Gemeinschaftsaufgabe (Bonn)

6) Jahreskolloquium: Ist die EG noch die angemessene Antwort auf die Herausforderungen unserer Zeit? (Bonn)

7) Entwicklungsmöglichkeiten für die Beziehungen zwischen Lateinamerika und der EG (Augsburg)

\section{4}

1) EG-Agrarpolitik im Wandel: Analyse und Bewertung der Vorschläge der EG-Kommission vom 28. Juli 1983 (Bonn)

2) Die Mittelmeerpolitik der EG unter Berücksichtigung der Süderweiterung (Lambrecht)

3) Die Durchsetzung des Gemeinschaftsrechts in den Mitgliedstaaten (Hamburg)

4) Umweltverträglichkeitsprüfung als europäisches Problem (Kaiserslautern)

5) EG und GATT (Bielefeld, Band 22)

6) Die Steuerharmonisierung in der EG (Hauenstein)
7) Sozialpolitik in der EG (Augsburg, Band 23)

8) Jahreskolloquium: Protektionistische Strömungen in der Weltwirtschaft und ihre Bedeutung für die EG-Außenhandelspolitik (Bremen, Band 24)

9) Fachtagung für Bibliothekare und Dokumentaristen der Europäischen Dokumentationszentren, Depositarbibliotheken und Referenzzentren (Köln)

\section{3}

\section{(502 Teilnehmer insgesamt)}

1) Die Versicherungswirtschaft im Gemeinsamen Markt (Ludwigshafen)

2) Probleme der wirtschaftlichen Beziehungen zwischen der EG und Japan (Feldkirchen-Westerham)

3) Probleme der Agrarstrukturpolitik der EG (Lambrecht)

4) Der Europäische Gerichtshof als Verfassungsgericht und Rechtsschutzinstanz (Hamburg, Band 20)

5) Integrationswirkungen von Verkehrssystemen und ihre Bedeutung für die EG (Augsburg)

6) Wirtschaftliche Integration in Europa während des 19. und 20. Jahrhunderts (Rauisch-Holzhausen)

7) Die Wettbewerbspolitik der EG (Hamburg)

8) Jahreskolloquium: Die Beziehungen zwischen der EG und den Vereinigten Staaten (Brüssel, Band 21)

9) Das Europäische Parlament vor der Herausforderung der ersten Wiederwahl (Bonn)

10) Neuorientierung in den Beziehungen zwischen der EG und den Entwicklungsländern (Berlin)

\section{2}

1) Die gefährdete Kohäsion der Gemeinschaft (Bornheim)

2) Fachtagung für die Leiter der Europäischen Dokumentationszentren, Depositarbibliotheken und Referenzzentren (Köln)

3) Die Rolle der EG bei der internationalen Versorgung mit landwirtschaftlichen Erzeugnissen (Lambrecht)

4) Die Beziehungen zwischen der EG und Lateinamerika-Bestandsaufnahme und Perspektiven (Berlin, Band 16)

5) Hörfunk und Fernsehen im Gemeinsamen Markt (Hamburg, Band 17)

6) Probleme des Finanztransfers über den EG-Haushalt (Bad Dürkheim)

7) Innergemeinschaftliche Machtungleichgewichte und die Rolle kleinerer Staaten (Günzburg)

8) Europäische Integration in der Lehrerausbildung an Hochschulen (Bocholt)

9) Gründung und Entwicklung der EG 1955-1966: Erfahrungen und Perspektiven (Köln)

10) Krisenvorsorge und Krisenmanagement in der EG (Augsburg, Band 18) 
11) Jahreskolloquium: Integrationskonzepte auf dem Prüfstand (Augsburg, Band 19)

12) Transnationale Politik in Europa: Der Beitrag von Verbänden zur europäischen Zusammenarbeit (Frankfurt/Main)

\section{1}

(248 Teilnehmer insgesamt, nur Tagungen 1-6)

1) Optionen einer Reform der EG-Agrarmarktpolitik

2) Europäisches Währungssystem - Erfahrungen und Perspektiven

3) Der Europäische Rat

4) Verbraucherschutz im Gemeinsamen Markt

5) Europäisches Verwaltungsrecht im Werden (Hamburg, Band 14)

6) Das Verhältnis zwischen der EG und den OPECStaaten

7) Jahreskolloquium: Politische Grundströmungen im europäischen Integrationsprozess (Köln, Band 15)

8) Das Problem einer Mitgliedschaft Spaniens in der EG

\section{0}

\section{(230 Teilnehmer insgesamt)}

1) Der Beitrag des Gerichtshofes der EG zur Verwirklichung des Gemeinsamen Marktes (Würzburg, Band 11)

2) Gespräch mit Europäischen Depositarbibliotheken, Dokumentations- und Referenzzentren

3) Innerdeutscher Handel zwischen deutschlandpolitischer Funktion und EG-Interessen

4) Aspekte der Politik der EG gegenüber der Dritten Welt

5) Aktivitäten von Interessenverbänden in der EG und ihre Rolle im Integrationsprozess

6) Die Zukunft des Budgets der EG

7) Jahreskolloquium: Industrie- und Strukturpolitik in der EG (Augsburg, Band 13)

8) Das Bankwesen in der EG (Glashütten, Band 12)

9) Der Beitrag der EG zum internationalen Interessenausgleich

\section{9}

\section{(215 Teilnehmer insgesamt, nur Tagungen 1-5)}

1) Integration und Kooperation im europäischen Währungswesen (Luxemburg, Band 8)

2) Neue Entwicklungen von Integrationstheorien

3) Die Interessenlagen westeuropäischer Staaten im europäischen Einigungsprozess - zeitgeschichtliche Analysen zum Zeitraum 1948-1958

4) Die EG als Rechtsgemeinschaft
5) Die Wahlen zum Europäischen Parlament im Rückblick

6) Jahreskolloquium: Arbeitsmarkt und Beschäftigungspolitik in der EG (Augsburg, Band 10)

\section{8}

(340 Teilnehmer insgesamt, nur Tagungen 1-8)

1) Die Beziehungen zwischen der EG und den Mitgliedstaaten des Rates für gegenseitige Wirtschaftshilfe

2) Die Rolle der gesellschaftlichen Kräfte in der EG

3) Die wirtschaftliche Nutzung der Nordsee und die EG (Hamburg, Band 6)

4) Öffentliche Aufträge und Forschungspolitik (Band 3)

5) Stand und Perspektiven der Curriculum-Entwicklung zur europäischen Integration für Hochschule und Schule

6) Bildungspolitik in der EG - Aktuelle Probleme und Ausblick (Berlin, Band 7)

7) Die EG nach einer neuen Erweiterungsrunde

8) Stand und Perspektiven der Assoziation EG-Türkei

9) Jahreskolloquium: Die Kernenergie als Problem europäischer Politik (Karlsruhe, Band 5)

10) Souveränitätsverständnis in den Europäischen Gemeinschaften (Otzenhausen, Band 9)

$$
1977
$$

(rund 240 Teilnehmer insgesamt, nur Tagungen 1-7)

1) Die europäische politische Zusammenarbeit (EPZ) - Ansatz zu einer Außenpolitik der Neun?

2) Die Erweiterung der EG nach Süden

3) Die Mittelmeerpolitik der EG

4) Die Grundrechte in den EG (Band 2)

5) Struktur- und regionalpolitische Probleme der europäischen Wirtschaftspolitik

6) Die Direktwahl zum europäischen Parlament

7) Willensbildungs- und Entscheidungsprozesse in den EG

8) Jahreskolloquium: Die EG im weltpolitischen und weltwirtschaftlichen Kräftefeld (Brüssel, Band 4)

\section{6}

(rund 180 Teilnehmer insgesamt, nur Tagungen 1-5)

1) Informationstagung für Hochschulassistenten (Königstein/Taunus)

2) Fachtagung für Politologen (Tübingen)

3) Interdisziplinäre Tagung für Hochschulassistenten (Berlin) 
4) Kolloquium für Juristen (Luxemburg)

5) Symposium für Sozialwissenschaftler und Bildungspolitiker (Bonn)

6) Die umstrittene Legitimität der EG (Bergisch Gladbach)

7) Friedenssicherung in Europa (Mannheim)

8) Jahreskolloquium: Wirtschafts- und gesellschaftspolitische Ordnungsprobleme der EG (Köln, Band 1)

\section{5}

1) Landwirtschaft in Europa - Aktuelle Probleme des Agrarrechts (Göttingen, 86)

2) Probleme politischer Planung in den EG (Darmstadt, 43)

3) Aktuelle Fragen der europäischen Integration (Bergisch Gladbach, 34)

4) Wechselwirkungen zwischen Raumordnung und Verkehr in der EG (München)

5) Jahreskolloquium: Ein neuer Auftrag für die EG: Gemeinsame Bewältigung von Krisen (Brüssel)

\section{4}

1) Interdependenz von Wirtschaft und Politik am Beispiel der EG (Bonn, 25)

2) Die Gemeinschaft in der Krise - Ursachen und Lösungsansätze (Hamburg, 56)

3) Energiepolitik der EG (Bielefeld, 55)

4) Theorien zur Integration (Baden bei Wien, 68)

5) Industrie- und Wettbewerbspolitik der Gemeinschaft - Gemeinsamkeiten und Widersprüche (Aachen, 46)

6) Jahreskolloquium: Die Beziehung der EG zu den Ländern der Dritten Welt (Berlin, 68)

7) Sozialpolitische Initiativen der EG (Düsseldorf, 55)

\section{3}

1) Entwicklungsperspektiven der Wirtschafts- und Währungsunion (Bergisch Gladbach, 44)

2) Aktuelle Fragen der europäischen Energiepolitik (Berlin, 21)

3) Die regionalpolitischen Probleme der erweiterten Gemeinschaft (Bonn, 31)

4) Probleme der Freizügigkeit der Arbeitnehmer in Europa (Darmstadt, 26)

5) Die Umweltschutzpolitik der EG (Mannheim, 45)

6) Jahreskolloquium: Die Außenwirtschaftsbeziehungen zwischen der EG und den Mitgliedsländern des Rates für gegenseitige Wirtschaftshilfe (Mainz)
1972

1) Aktuelle Fragen der europäischen Integration (Berlin, 78)

2) Fachtagung für Juristen (Würzburg, 54)

3) Fachtagung für Politologen (Bonn, 21)

4) Agrarstruktur- und Regionalpolitik der EG (Königstein/Taunus, 40)

5) Die EG gegenüber der Dritten Welt (Springen/Taunus, 51)

6) Jahreskolloquium: Die Außenbeziehungen der erweiterten Gemeinschaft (Brüssel, 72)

7) Wachstumszyklen und Konjunkturpolitik in der EWG (Bonn, 40)

\section{1}

1) Aktuelle Fragen der europäischen Integration (Speyer, 73)

2) Sozialpolitik in der EG (Springen/Taunus, 29)

3) Fachtagung für Politologen (Bonn, 23)

4) Zielvorstellungen der europäischen Einigung (Speyer, 40)

5) Verfassungsprobleme der EG (Springen/Taunus, 38)

6) Jahreskolloquium: Strukturfragen der europäischen Einigung (Bonn, 67)

7) Fachtagung für Politologen (Bonn, 30)

\section{0}

1) Die EG in der Endphase (Berlin, 76)

2) Die Weiterführung der europäischen Integration (Bonn, 20)

3) Die institutionelle Struktur der EG (Bergisch Gladbach, 40)

4) Integration und Währungsunion (Bonn, 82)

5) Jahreskolloquium: Strukturfragen der europäischen Einigung (Bonn, 68)

6) Die politische Problematik des europäischen Integrationsprozesses (Saarbrücken, 29)

7) Die Interdependenz der Volkswirtschaften und die wirtschaftspolitische Programmierung in der EG (Bonn, 55)

\section{9}

1) Professorenkolloquium

2) Informationsseminar (Königstein/Taunus)

3) Politologen-Tagung (Bonn)

4) Berlin-Tagung

5) Assistentenseminar (Bonn)

6) Assistentenseminar (Bonn)

7) Politologen-Tagung (Bonn) 


\section{Anhang III: Die Schriftenreihe des AEI**}

Grundfragen und Grundstrukturen der europäischen Integration

- Wirtschafts- und gesellschaftspolitische Ordnungsprobleme der Europäischen Gemeinschaften (AEI-Vorstand, Bd. 1, 1978)

- Souveränitätsverständnis in den Europäischen Gemeinschaften (Georg Ress, Bd. 9, 1980)

- Politische Grundströmungen im europäischen Integrationsprozess (AEI-Vorstand, Bd. 15, 1982)

- Integrationskonzepte auf dem Prüfstand (AEI-Vorstand, Bd. 19, 1983)

- Deutsch-deutsche Wirtschafts-, Währungs- und Sozialunion im Rahmen der EG (Wulfdiether Zippel, Bd. 30, 1991)

- Die Entwicklung der EG zur Politischen Union und zur Wirtschafts- und Währungsunion unter der Sonde der Wissenschaft (Rudolf Hrbek, Bd. 32, 1993)

- Der Vertrag von Maastricht in der wissenschaftlichen Kontroverse (Rudolf Hrbek, Bd. 34, 1993)

- Bürger und Europa (Rudolf Hrbek, Bd. 35, 1994)

- Die Reform der EU (Rudolf Hrbek, Bd. 41, 1997)

- Gemeinsames Verfassungsrecht in der EU (Peter-Christian Müller-Graff/Eibe Riedel, Bd. 43, 1998)

- Solidarität und Beitragsgerechtigkeit (Ines Hartwig/Wolfgang Petzold, Bd. 53, 2005)

- Deutschlands Rolle in der EU (Peter-Christian Müller-Graff, Bd. 60, 2008; 2. Aufl. 2009)

- Nachbarschaften innerhalb der EU (Ulrich Hufeld/Peter-Christian Müller-Graff/Stefan Okruch, Bd. 62, 2007)

- Der Zusammenhalt Europas - In Vielfalt geeint (Peter-Christian Müller-Graff, Bd. 64, 2009)

- Die EU-Reflexionsgruppe „Horizont 2020-2030“ (Gabriele Abels/Annegret Eppler/Michèle Knodt, Bd. 69, 2010)

- Debating Europe (Robert Harmsen/Joachim Schild, Bd. 71, 2011)

- Polen als Motor des europäischen Integrationsprozesses (Beate Neuss/Antje Nötzold, Bd. 77, 2013)

- Zur Konzeptualisierung europäischer Desintegration (Annegret Eppler/Henrik Scheller, Bd. 78, 2013)

- The EU between an ,Ever Closer Union“ and Inalienable Policy Domains of Member States (Thomas Giegerich/Oskar J. Gstrein/Sebastian Zeitzmann, Bd. 80, 2014)

\section{Institutionen der EG/EU}

- Der Europäische Gerichtshof als Verfassungsgericht und Rechtsschutzinstanz (Jürgen Schwarze, Bd. 19, 1983)

- Das Europäische Parlament (Andreas Maurer/Dietmar Nickel, Bd. 54, 2005)

- Auf dem Weg zum Mehrebenenparlamentarismus? (Gabriele Abels/Annegret Eppler, Bd. 75, 2011)

\footnotetext{
** In Klammern finden sich jeweils die Herausgeber, die Nummer des Bandes in der Schriftenreihe des AEI sowie das Erscheinungsjahr.
} 
Kernbereich der europäischen Integration (gemeinsamer Binnenmarkt, Wirtschafts- und Währungsunion)

- Die Kernenergie als Problem europäischer Politik (AEI-Vorstand, Bd. 5, 1980)

- Die wirtschaftliche Nutzung der Nordsee und die EG (Hans R. Krämer, Bd. 6, 1979)

- Integration und Kooperation im Europäischen Währungswesen (Hugo J. Hahn, Bd. 8, 1980)

- Arbeitsmarkt und Beschäftigungspolitik (AEI-Vorstand, Bd. 10, 1981)

- Der Beitrag des Gerichtshofes der EG zur Verwirklichung des Gemeinsamen Marktes (ErnstWerner Fuß, Bd. 11, 1981)

- Das Bankwesen im Gemeinsamen Markt (Uwe Blaurock, Bd. 12, 1981)

- Industrie- und Strukturpolitik in der EG (AEI-Vorstand, Bd. 13, 1981)

- Hörfunk und Fernsehen im Gemeinsamen Markt (Martin Seidel, Bd. 17, 1983)

- Die soziale Dimension des Europäischen Binnenmarktes (Rolf Birk, Bd. 27, 1990)

- Technische Regeln im Binnenmarkt (Peter-Christian Müller-Graff, Bd. 28, 1991)

- Der Schutz des geistigen Eigentums in Europa (Meinhard Hilf/Wolfgang Oehler, Bd. 29, 1991)

- Transeuropäische Netze (Wulfdiether Zippel, Bd. 39, 1996)

- Zur Reform der Finanzverfassung und Strukturpolitik der EU (Rolf Caesar, Bd. 42, 1997)

- Kommunale Aufgaben im Binnenmarkt (Oscar Gabriel/Peter-Christian Müller-Graff/Christian O. Steger, Bd. 68, 2010)

- Europäisierte Regulierungsstrukturen und -netzwerke (Ludwig Gramlich/Cornelia MangerNestler, Bd. 74, 2011)

\section{Flankierende Politikfelder der europäischen Integration}

- Die Grundrechte in der EG (Jochen Frowein, Bd. 2, 1978)

- Öffentliche Aufträge und Forschungspolitik (Karl M. Meessen, Bd. 3, 1979)

- Bildungspolitik in der EG (Hermann Schmitz-Wenzel, Bd. 7, 1980)

- Europäisches Verwaltungsrecht im Werden (Jürgen Schwarze, Bd. 14, 1982)

- Krisenvorsorge und Krisenmanagement in der EG (Manfred Zuleeg, Bd. 18, 1984)

- Sozialpolitik in der EG (Hagen Lichtenberg, Bd. 23, 1986)

- Ausländerrecht und Ausländerpolitik in Europa (Manfred Zuleeg, Bd. 26, 1987)

- Gemeinsames Privatrecht in der EG (Peter-Christian Müller-Graff, Bd. 33, 1993; 2. Aufl. 1999)

- Das Subsidiaritätsprinzip in der EU (Rudolf Hrbek, Bd. 36, 1995)

- Frauenpolitik in der EU (Melanie Piepenschneider, Bd. 37, 1996)

- Europäische Zusammenarbeit in den Bereichen Justiz und Inneres (Peter-Christian MüllerGraff, Bd. 38, 1996)

- Sozialstaatliche Entwicklungen in Europa und die Sozialpolitik der EU (Hans-Wolfgang Platzer, Bd. 40, 1997)

- Studying Europe (Hagen Lichtenberg/Wolfgang Petzold, Bd. 45, 1999)

- Arbeitsmarkt- und Beschäftigungspolitik in der EU (Hans-Wolfgang Platzer, Bd. 46, 1999)

- Kirchen und Religionsgemeinschaften in der EU (Peter-Christian Müller-Graff/Heinrich Schneider, Bd. 50, 2003)

- Der Raum der Freiheit, der Sicherheit und des Rechts (Peter-Christian Müller-Graff, Bd. 51, 2005)

- Die Gleichstellungspolitik in der EU (Annette Jünemann/Carmen Klement, Bd. 52, 2005)

- Medien in der EG (Dieter Dörr/Peter-Christian Müller-Graff, Bd. 59, 2007)

- Europäisches Flüchtlings- und Einwanderungsrecht (Rainer Hoffmann/Tillmann Löhr, Bd. 63, 2008) 
- Europäisches Gesellschaftsrecht auf neuen Wegen (Peter-Christian Müller-Graff/Christoph Teichmann, Bd. 67, 2010)

- International Anti-Corruption Regimes in Europe (Sebastian Wolf/Diana Schmidt-Pfister, Bd. $70,2010)$

\section{Beziehungen der EG/EU zu anderen Staaten und Regionen}

- Die EG im weltpolitischen und weltwirtschaftlichen Kräftefeld (AEI-Vorstand, Bd. 4, 1979)

- Die Beziehungen zwischen der EG und Lateinamerika (Hans J. Petersen, Bd. 16, 1983)

- Die Beziehungen zwischen der EG und den Vereinigten Staaten (AEI-Vorstand, Bd. 21, 1984)

- EG und Drittstaatsbeziehungen nach 1992 (Meinhard Hilf/Christian Tomuschat, Bd. 31, 1991)

- Die Mittelmeerpolitik der EU (Wulfdiether Zippel, Bd. 44, 1999)

- Die Beziehungen zwischen der EU und den Mercosur-Staaten (Wulfdiether Zippel, Bd. 48, 2001)

- Spezifika einer Südost-Erweiterung der EU (Wulfdiether Zippel, Bd. 49, 2003)

- Die Schweiz im europäischen Integrationsprozess (Fritz Breuss/Thomas Cottier/Peter-Christian Müller-Graff, Bd. 61, 2008)

- Osteuropäische Nachbarschaft der EU (Kazimierz Lankosz/Peter-Christian Müller-Graff/Udo Fink, Bd. 65, 2009)

- Turkey and the EU (Peter-Christian Müller-Graff/Haluk Kabaalioglu, Bd. 76, 2012)

Allgemeine Außen-, Sicherheits- und Außenhandelspolitik der EG/EU

- GATT und EG (Meinhard Hilf/Ernst-Ulrich Petersmann, Bd. 22, 1986)

- Neuer Protektionismus in der Weltwirtschaft und EG-Handelspolitik (AEI-Vorstand, Bd. 24, 1985)

- Sicherheitspolitische Zusammenarbeit und Kooperation der Rüstungswirtschaft in Westeuropa (Lothar Brock/Mathias Jopp, Bd. 25, 1986)

- Die EG in der Welthandelsorganisation (Peter-Christian Müller-Graff, Bd. 47, 1999)

- Die Außenwirtschaftspolitik der EU nach dem Verfassungsvertrag (Christoph Herrmann/Horst G. Krenzler/Rudolf Streinz, Bd. 55, 2006)

- Die Rolle der erweiterten EU in der Welt (Peter-Christian Müller-Graff, Bd. 57, 2006)

- Externe Demokratieförderung durch die EU (Annette Jünemann/Michèle Knodt, Bd. 58, 2007)

- Europe and New Leading Powers (Jörg Husar/Günther Maihold/Stefan Mair, Bd. 66, 2010)

- The EU in International Fora (Julia Lieb/Nicolai von Ondarza/Daniela Schwarzer, Bd. 72, 2011)

- Die gemeinsame Handelspolitik der EU nach Lissabon (Marc Bungenberg/Christoph Herrmann, Bd. 73, 2011)

- Grenzen der Integration (Eckart D. Stratenschulte, Bd. 79, 2013) 